\title{
Percepciones institucionales de los estudiantes de Pedagogía en Educación Física de Chile Institutional perceptions of Physical Education Pedagogy students from Chile
}

*Elizabeth Flores Ferro, **Fernando Maureira Cid, ***Ángela Silva-Salse, *Marcelo Muñoz Lara, *Alexis Matheu Pérez, *Braulio Navarro Aburto, ****Valentina Bahamondes Acevedo, *****Marcelo Hadweh Briceño, ******Juan Hurtado Almonacid, *****Michel Valdés-Montecinos, *******Esteban Aedo Muñoz, ********Sebastián Peña-Troncoso, *********Daniel Serey Araneda, **********Juan Pablo Zavala-Crichton, ***********Juan Maureira Sánchez, ***********Marcela Brevis-Yeber, *Mario Valero Catalán

*Universidad Bernardo O’Higgins (Chile), **Universidad Católica Silva Henríquez (Chile), ***Universidad SEK (Chile),

****Universidad Metropolitana de Ciencias de la Educación (Chile), *****Universidad Arturo Prat Santiago (Chile), ******Pontificia Universidad Católica de Valparaíso (Chile), *******Universidad Metropolitana de Ciencias de la Educación (Chile)/ Universidad de Santiago de Chile (Chile), ********Universidad Austral de Chile (Chile), *********Universidad de Atacama (Chile), **********Universidad Andres Bello (Chile), ***********Universidad Central Región de Coquimbo (Chile)

Resumen. Conocer la percepción institucional de los agentes que componen una comunidad resulta relevante para mejorar los servicios educativos que se prestan, además permiten instaurar mecanismos de seguimiento, control e innovación dentro de las universidades para lograr una mejora continua. El objetivo de la presente investigación fue determinar la percepción institucional de los estudiantes de Pedagogía en Educación Física de Chile. Se aplicó la escala de percepción hacia la carrera educación física a 908 estudiantes, de los cuales 317 eran mujeres (34.9\%) y 591 hombres (65.1\%). El promedio de edad fue de $20.1 \pm 2.4$ y 359 eran de primer año, 316 de segundo y 233 de tercer año de diferentes instituciones de educación superior de Chile. Se encontraron diferencias significativas al comparar las universidades privadas con las públicas (percepción favorable hacia las instituciones privadas) en la gestión universitaria $(p=.000)$ y protestas estudiantiles $(p=.000)$; por otro lado, también se puede enfatizar que los estudiantes perciben que les hace falta conocer técnicas de estudio, sobre todo los alumnos provenientes de colegios municipales $(p=.049)$ en relación con los establecimientos particulares. Se concluye que la percepción institucional de los estudiantes de pedagogía en educación física que tiene mayor relevancia es la gestión académica. Son necesarias futuras investigaciones con otras variables y de corte longitudinal.

Palabras Claves: percepción, educación física, gestión universitaria, protestas estudiantiles.

Abstract. Knowing the institutional perception of the agents building up a community is important to improve educational services provided, and allows for the establishment of monitoring, control, and innovation mechanisms within universities to achieve continuous improvement. The objective of this research was to determine the institutional perception of students of Pedagogy in Physical Education from Chile. A total of 908 students ( 317 women [34.9\%]; and 591 men [65.1\%]) with average age of $20.1 \pm 2.4$ years old, enrolled in firstyear (359), second year (316), and third year (233) at different higher education institutions in Chile filled the Perception of Physical Education Degree Scale. Results: Significant differences were found comparing private and public universities (private institutions showing more favorable perceptions) in university management $(\mathrm{p}=.000)$ and students' protests $(\mathrm{p}=.000)$; on the other hand, it can also be emphasized that students perceive that they need to know study techniques, especially students from municipal schools $(\mathrm{p}=.049)$ in relation to private institutions. As a conclusion, the most relevant institutional perception of students of pedagogy in physical education regards academic management. Future research with other variables and longitudinal character is necessary.

Keywords: Perception, physical education, university management, student protests.

\section{Introducción}

En los últimos años las instituciones de educación superior se han sometido a procesos de evaluación debido a la necesidad de reconocimiento y acreditación por parte de entidades externas (López, 2007). Las universidades se han preocupado por los factores relacionados con la percepción positiva, de parte de los estudiantes y docentes, en cuanto al servicio de calidad educativa que ofrecen; actualmente, conocer la percepción de los alumnos es fundamentales para las instituciones de educación superior, ya que con ello se puede planificar, controlar, realizar seguimientos y mejorar las funciones que se están realizando en la universidad (Reyes y Reyes, 2012). Cervantes, Peralta, Salgado \& Stefanell (2018) agregan que los administradores de nivel superior tienen la responsabilidad de evaluar, implementar y proponer estrategias para mejorar la oferta de servicios de su organismo.

Fecha recepción: 25-11-19. Fecha de aceptación: 02-02-20
Una de las áreas más estudiadas sobre la percepción institucional, se relaciona con la calidad de servicio percibida por parte de los alumnos universitarios (en este contexto hace referencia a los estándares de formación de profesionales) donde a menudo se comparan las universidades públicas versus las privadas (Alvarado, Luyando y Picazzo, 2015). En este sentido, Camisón, Gil y Roca (1999) destacan la preparación técnica del profesorado, el acceso y la infraestructura que son rasgos importantes en educación. Capelleras y Veciana (2004) suman a lo anterior, los contenidos de los planes de estudio, las actitudes de los funcionarios, el equipamiento y la organización de la enseñanza. Por otro lado, Sarmiento y Vinueza (2020) agregan que también deben considerarse la comodidad y conocimiento, ya que estas son bien valoradas y se relacionan con la gestión académica y administrativa de las instituciones de educación superior.

Una investigación de Sotomayor, Coloma, Parodi, Ibáñez, Cavada y Gysling (2013) analizó la percepción de estudiantes de pedagogía en educación básica sobre su plan de estudios, destacándose la valoración en la formación recibida, asignándole más importancia al ámbito práctico que al teóri- 
co. Por otra parte, un estudio de Bustamante, Lapo, Oyarzún y Campos (2017) sobre el desempeño docente, muestra que los estudiantes perciben una alta participación, personalización de la enseñanza, motivación, desarrollo de autoestima e interacción por parte de sus profesores universitarios. En relación con la percepción hacia la carrera de pedagogía en educación física se puede mencionar el trabajo de Cea, Véliz, Aravena y Maureira (2014) donde mostraron que los estudiantes de cursos mayores poseen una percepción menos positiva, debido a una visión más crítica que poseen por su formación académica. Otro estudio que apoya estos resultados es el de Poblete, Linzmayer, Matus, Garrido y Flores (2018) donde también evaluaron a estudiantes de educación física de Chile encontrando que a medida que los alumnos cursan niveles más altos se vuelven más críticos.

Otro elemento fundamental dentro de las instituciones de educación superior corresponde a la formación académica de los estudiantes, para el plan de estudios de pedagogía en educación física Moreno, Sicilia y Águila (2003) afirman que existen dos modelos en la disciplina, a) pedagógica: que corresponde al rol docente y su relación con los procesos de enseñanza y aprendizaje, b) especializada: más dirigida a los conocimientos específicos y dominios desde el aprendizaje motor. Gutiérrez, Domenéc y Torres (2007) postulan que la formación del profesor de educación física debe obedecer a cómo ser buen docente y cómo debe evaluarse las competencias de estos profesionales de la actividad física.

Por otro lado, los motivos que llevan a los estudiantes para elegir una carrera se ven influenciadas por el ámbito social, afectivo y experiencias previas de los sujetos, todo ello se puede interpretar como vocación, lo cual es relevante para la formación profesional de los estudiantes y lamentablemente poco estudiada en la disciplina de la educación física (Mujica y Orellana, 2016). En este sentido, García (2019) plantea que la vocación es relevante para que el estudiante desde temprana edad se identifique profesionalmente, considera que es un elemento que inicia desde los primeros años académicos.

En un estudio realizado por Flores, Bahamondes, Maureira y González (2019) construyeron y validaron un instrumento para conocer la percepción institucional de estudiantes de educación física de Chile. Los autores muestran que estos alumnos consideran que la exigencia académica es alta, que tienen buenas relaciones sociales y que están seguros de su vocación. Otro estudio de Urra, Reyno, Fehrenberg y Muñoz (2020) evaluaron la percepción del rol docente en estudiantes de pedagogía en educación física de Chile, mostrando la relevancia de las experiencias previas, la valoración de las asignaturas cursadas y también identificaron atributos personales por sobre elementos metodológicos, conceptuales y valóricos para caracterizar a un buen docente. En esta línea, Urra, Fehrenberg, Muñoz, Matheu y Reyno (2019) mostraron resultados similares, agregando que existe una valoración positiva de la clase de educación física en relación con los contenidos y actividades previamente experimentadas.

En base a lo expuesto, resulta fundamental conocer la percepción de los agentes que componen una comunidad educativa, ya que con ello incluso se puede prevenir los niveles de deserción en cualquier disciplina e ir mejorando constantemente para ser una institución de educación superior de excelencia. En consecuencia, se plantea el siguiente objetivo de investigación: determinar la percepción institucional de los estudiantes de pedagogía en educación física de Chile.

\section{Material y método}

\section{Tipo de investigación}

El presente estudio fue de tipo cuantitativo, de corte transversal y con un diseño no-experimental (Maureira y Flores, 2018).

\section{Muestra}

Constituida por 908 estudiantes que cursaban pedagogía en educación física durante el segundo semestre del año académico 2019, de los cuales 317 eran mujeres (34.9\%) y 591 hombres (65.1\%). La edad mínima fue de 18 y la máxima de 45 con una media de 20.1 \pm 2.4 . 359 estudiantes de primer año (39.5\%), 316 de segundo año (34.8\%) y 233 de tercer año (25.7\%). Los estudiantes de instituciones públicas fueron: 129 de la U. metropolitana de Ciencias de le Educación (14,2\%) y 48 a la U. de Atacama (5.3\%). En cuanto a las instituciones privadas, 62 estudiantes pertenecen a la U. Central región de Coquimbo (6.8\%), 103 a la U. Austral de Chile (11.3\%), 85 a la U. Andrés Bello, Viña del Mar (9.4\%), 180 a la U. Católica de Valparaíso (19.8\%), 39 a la U. Bernardo O'Higgins (4.3\%), 34 a la U. Autónoma de Temuco (3.7\%) y 228 a la U. católica Silva Henríquez (25,1\%).

\section{Instrumento}

Se utilizó la escala de Percepción de la carrera de pedagogía en Educación Física (Flores, et al., 2019), el instrumento consta de 20 ítems en formato Likert con 5 opciones, siendo el nivel 1 muy en desacuerdo y el nivel 5 muy de acuerdo. El instrumento fue validado en estudiantes de Educación Física de Chile.

La escala administrada presentó un alfa de Cronbach de .672 y el análisis factorial exploratorio entregó siete dimensiones que explican el 55,8\% de la varianza:

1. Gestión universitaria, con los siguientes ítems: Existe buena organización institucional (2); Los docentes tienen un alto nivel académico (3); El programa de la carrera responde a mis expectativas (4); Los programas por asignaturas responden a mis expectativas (5); Si tuviera la oportunidad me cambiaría a otra universidad a la misma carrera (15); La universidad cumple con los compromisos adquiridos con los estudiantes (19) y La universidad ha mejorado en el último tiempo en infraestructura y docencia (20).

2. Apoyo social y familia, con los ítems: Siento apoyo familiar en la carrera que elegí (16) y Mi entorno social considera que escogí una buena carrera (17).

3. Exigencia de la carrera, con los ítems: El nivel de exigencia académica es alta (1); La exigencia de la carrera afecta a mi salud psicológica (9) y La exigencia de los ramos deportivos afecta a mi salud física (10).

4. Financiamiento y formación con los ítems: Pienso que mi formación de colegio es alta respecto a mis compañeros (12); Mis horarios son compatibles con mi trabajo laboral 
(14) y Actualmente puedo financiar económicamente mi carrera (18).

5. Protestas estudiantiles con los ítems: Considero que los paros son extensos en mí institución (6) y Me genera estrés post paro la acumulación de pruebas, trabajos, informes, etc. (8).

6. Participación y nivelación con los ítems: Me siento participe de las decisiones del centro de alumnos (7) y La institución cuenta con cursos de nivelación (13).

7. Técnicas de estudio con el ítem: Pienso que me hace falta conocer técnicas de estudio (11).

Se debe enfatizar que los ítems $1,6,8,9,10,11$ y 15 se codificaron en forma inversa.

\section{Procedimiento}

La recolección de datos fue realizada en forma grupal y durante las horas de clases a principio del segundo semestre (entre agosto y septiembre del año 2019). Cada estudiante que cursaba educación física tuvo la opción de participar o no en la investigación si lo deseaba. Cada sujeto que conformó la muestra firmó un consentimiento informado.

\section{Análisis estadístico}

Se utilizó el programa estadístico SPSS 25.0 para Windows. Se utilizó pruebas KS de normalidad donde se obtuvieron valores $\mathrm{p}<.005$ en todas las dimensiones, por lo que se procedió a utilizar estadística paramétrica. Para comparar las dimensiones por sexo, con o sin estudios previos y tipo de universidad se utilizó prueba $t$ para muestras independientes; y para comparar entre las siete dimensiones, con el año académico, ingreso familiar, tipo de colegio se utilizó la prueba de ANOVA (Maureira, 2017). Se consideró significativo un valor $p<.05$.

\section{Resultados}

En la tabla 1 se puede observar que sólo se encontraron diferencias significativas en la dimensión Protestas estudiantiles, dónde se puede interpretar que las mujeres sienten mayor estrés cuando la institución se va a paro y a su vez consideran estas protestas más extensas que los hombres de la muestra.

También se realizó una prueba de ANOVA para comparar las siete dimensiones del total de la muestra, encontrando diferencias significativas entre ellas $(F=123.749$; $g l=6$; $p=.000$ ), donde la dimensión tres que se refiere a la Exigencia de la carrera posee mejor valoración y las dimensiones que tienen más bajas valoraciones son la Gestión universitaria, Apoyo social y familiar; y por último Protestas estudiantiles.

\begin{tabular}{|c|c|c|c|c|c|}
\hline Dimensión & $\begin{array}{c}\text { Total } \\
(\mathrm{n}=908)\end{array}$ & Mujeres $(\mathrm{n}=31$ & ombres $(\mathrm{n}=591)$ & $p$ & $d$ \\
\hline Gestión universitaria & $3.8 \pm .67$ & $3.8 \pm .64$ & $3.8 \pm .68$ & .921 & \\
\hline Apoyo social y familiar & $4.2 \pm .95$ & $4.2 \pm .99$ & $4.2 \pm .94$ & .726 & \\
\hline Exigencia de la carrera & $3.3 \pm .77$ & $3.3 \pm .75$ & $3.3 \pm .75$ & .381 & \\
\hline Financiamiento y formación & $3.2 \pm .87$ & $3.2 \pm .87$ & $3.2 \pm .87$ & .638 & \\
\hline Protestas estudiantiles & $2.7 \pm 1.18$ & $2.5 \pm 1.14$ & $2.8 \pm 1.19$ & $.003 * *$ & .28 \\
\hline Participación y nivelación & $2.7 \pm .95$ & $2.7 \pm 1.00$ & $2.7 \pm .93$ & .705 & \\
\hline Técnicas de estudio & $2.3 \pm 1.20$ & $2.3 \pm 1.19$ & $2.3 \pm 1.18$ & .795 & \\
\hline
\end{tabular}

En la tabla 2 se puede observar las comparaciones de las medias por dimensión según año que cursaban los estudiantes de Educación Física, se puede apreciar que en las dimensiones $1,3,5$ y 7 presentan diferencias significativas, donde de manera global se puede interpretar que a medida que los alumnos reciben su formación académica, estos se vuelven más críticos en la percepción de la dimensión Gestión universitaria. En cuanto a la dimensión Exigencia de la Carrera, los estudiantes de primer y segundo año consideran más exigente su formación (en relación a lo físico, psicológico y académico) que los estudiantes de tercer año. Por otra parte, los estudiantes de primer año consideran que las Protestas estudiantiles les afectan más y consideran que la duración de estos es más extensa que los estudiantes de segundo y tercer año. En relación con la dimensión de Técnicas de estudio, los estudiantes de segundo se perciben con mejores habilidades para estudiar que los estudiantes de primer año.

\begin{tabular}{|c|c|c|c|c|c|}
\hline Dimensión & $\begin{array}{c}1^{\circ} \text { año } \\
(\mathrm{n}=359)\end{array}$ & $\begin{array}{c}2^{\circ} \text { año } \\
(\mathrm{n}=316)\end{array}$ & $\begin{array}{c}3^{\circ} \text { año } \\
(\mathrm{n}=233)\end{array}$ & $p$ & Tukey \\
\hline Gestión universitaria & $3.9 \pm .69$ & $3.8 \pm .65$ & $3.7 \pm .64$ & $.000^{* *}$ & $1^{\circ}>2^{\circ}-3^{\circ}$ \\
\hline Apoyo social y familiar & $4.2 \pm 1.00$ & $4.2 \pm .92$ & $4.2 \pm .89$ & .800 & \\
\hline Exigencia de la carrera & $3.2 \pm .73$ & $3.3 \pm .74$ & $3.4 \pm .77$ & $.014^{*}$ & $3^{\circ}>1^{\circ}-2^{\circ}$ \\
\hline Financiamiento y formación & $3.1 \pm .83$ & $3.3 \pm .88$ & $3.2 \pm .91$ & .116 & \\
\hline Protestas estudiantiles & $2.9 \pm 1.26$ & $2.5 \pm 1.11$ & $2.5 \pm 1.09$ & $.000^{* *}$ & $1^{\circ}>2^{\circ}-3^{\circ}$ \\
\hline Participación y nivelación & $2.7 \pm .92$ & $2.6 \pm .94$ & $2.6 \pm 1.03$ & .343 & \\
\hline Técnicas de estudio & $2.2 \pm 1.19$ & $2.4 \pm 1.21$ & $2.2 \pm 1.19$ & $.023^{*}$ & $2^{\circ}>1^{\circ}$ \\
\hline
\end{tabular}

También se realizaron comparaciones de las dimensiones de la escala con las respuestas si tenían hijo o no, los análisis indican que no existen diferencias significativas en ninguna dimensión entre estudiantes que tienen $(\mathrm{n}=28)$ y no tienen hijos $(\mathrm{n}=877)$, por lo tanto, parece ser que esta condición no incide en la percepción positiva o negativa de la carrera de Educación Física.

En la tabla 3, se puede observar las comparaciones de las percepciones de los estudiantes con y sin estudios previos, se puede apreciar que existen diferencias significativas en las dimensiones Exigencia de la carrera y Técnicas de estudio, es decir, los que poseen estudios previos consideran que la carrera es menos exigente que los estudiantes que cursan por primera vez estudios superiores, y por otra parte, los alumnos que no poseen estudios previos consideran que les hace falta conocer técnicas de estudio en relación a las personas que ya cursaron otra carga académica.

\begin{tabular}{|c|c|c|c|c|}
\hline Dimensión & $\begin{array}{c}\text { Sin estudios previos } \\
(\mathrm{n}=715)\end{array}$ & $\begin{array}{c}\text { Con estudios previos } \\
(\mathrm{n}=189)\end{array}$ & $p$ & $d$ \\
\hline Gestión universitaria & $3.8 \pm .66$ & $3.8 \pm .69$ & .346 & \\
\hline Apoyo social y familiar & $4.2 \pm .94$ & $4.1 \pm .98$ & .210 & \\
\hline Exigencia de la carrera & $3.2 \pm .74$ & $3.5 \pm .76$ & $.000 * *$ & .35 \\
\hline Financiamiento y formación & $3.2 \pm .88$ & $3.2 \pm .86$ & .444 & \\
\hline Protestas estudiantiles & $2.6 \pm 1.16$ & $2.8 \pm 1.22$ & .124 & \\
\hline Participación y nivelación & $2.7 \pm .96$ & $2.6 \pm .97$ & .108 & \\
\hline Técnicas de estudio & $2.2 \pm 1.17$ & $2.5 \pm 1.31$ & $.002^{* *}$ & .27 \\
\hline
\end{tabular}

En la tabla 4 se puede observar que existen diferencias significativas en la dimensión Gestión universitaria, Financiamiento y formación, y Técnicas de estudio según tipo de establecimiento educacional, en la primera se puede apreciar que los estudiantes de colegios subvencionados consideran que su institución posee mejor gestión en relación con los alumnos provenientes de colegios municipales. En la dimensión cuatro, se puede interpretar que existe una 
gran diferencia entre los estudiantes provenientes de colegios municipales en comparación a los subvencionados y particulares, siendo estos últimos los que consideran que sus horarios y formación son más adecuados en relación con los que provienen de colegios municipales. Por otra parte, en la dimensión siete, los alumnos de colegios particulares consideran que poseen mejores habilidades para estudiar respecto a sus compañeros provenientes de colegios municipales y subvencionados.

Tabla 4

Pruebas ANOVA comparando los puntajes de cada dimensión según subvención de los establecimientos educacionales de origen.

\begin{tabular}{lccccc}
\hline \multicolumn{1}{c}{ Dimensión } & $\begin{array}{c}\text { Municipal } \\
(\mathrm{n}=259)\end{array}$ & $\begin{array}{c}\text { Subvencionado } \\
(\mathrm{n}=540)\end{array}$ & $\begin{array}{c}\text { Particular } \\
(\mathrm{n}=108)\end{array}$ & $p$ & Tukey \\
\hline Gestión universitaria & $3.7 \pm .65$ & $3.8 \pm .65$ & $3.8 \pm .78$ & $.019^{*}$ & $\mathrm{~S}>\mathrm{M}$ \\
Apoyo social y familiar & $4.1 \pm .93$ & $4.2 \pm .96$ & $4.3 \pm .91$ & .251 & \\
Exigencia de la carrera & $3.2 \pm .74$ & $3.3 \pm .74$ & $3.4 \pm .80$ & .062 & \\
Financiamiento y formación & $2.9 \pm .88$ & $3.3 \pm .85$ & $3.4 \pm .84$ & $.000^{* *}$ & $\mathrm{~S}-\mathrm{P}>\mathrm{M}$ \\
Protestas estudiantiles & $2.6 \pm 1.21$ & $2.8 \pm 1.17$ & $2.5 \pm 1.12$ & .055 & \\
Participación y nivelación & $2.6 \pm .98$ & $2.7 \pm .94$ & $2.5 \pm .95$ & .058 & \\
Técnicas de estudio & $2.3 \pm 1.18$ & $2.2 \pm 1.16$ & $2.5 \pm 1.44$ & $.049^{*}$ & $\mathrm{P}>\mathrm{M}-\mathrm{S}$ \\
\hline $\mathrm{M}=$ municipal; S=subvencionado; $\mathrm{P}=$ particular. & & & & \\
*Diferencias significativas al nivel .05 & & & & \\
***iferencias significativas al nivel .01 & & & &
\end{tabular}

En la tabla 5 se puede observar que existen diferencias significativas en cinco de las siete dimensiones del instrumento, donde cabe enfatizar que de manera global los resultados indican que a medida que se posee un mayor ingreso familiar también mejora la percepción de la institución educativa en donde estudian, excepto en la dimensión Apoyo Social y familiar, donde los estudiantes del G3 se considera más apoyados en relación a los que perciben mayor ingreso (G4) y en la dimensión Participación y nivelación donde el G4 percibe que hace falta más cursos de nivelación y que los consideren en la toma de decisiones del centro de alumnos.

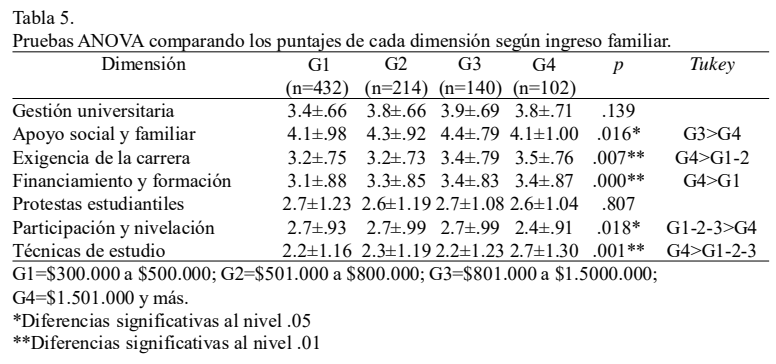

Finalmente, en la tabla 6 donde se compara los resultados de la muestra según tipo de universidad (pública y privada) y las dimensiones del instrumento, se puede observar que en tres de las siete dimensiones existen diferencias significativas, el primero Gestión universitaria, donde los estudiantes de las universidades estatales consideran bajo nivel en la gestión en relación a los alumnos de instituciones privadas, en la dimensión Protestas estudiantiles, también a favor de las universidades privadas y repitiéndose lo mismo en la dimensión Participación y nivelación también con mejor percepción de los estudiantes de las instituciones privada.

Tabla 6 .

Pruebas t comparando los puntajes de cada dimensión entre estudiantes de universidades estatales y privadas.

\begin{tabular}{lcccc}
\multicolumn{1}{c}{ Dimensión } & $\begin{array}{c}\text { U. estatales } \\
(\mathrm{n}=177)\end{array}$ & $\begin{array}{c}\text { U. privadas } \\
(\mathrm{n}=731)\end{array}$ & $p$ & $d$ \\
\hline Gestión universitaria & $3.4 \pm .56$ & $3.9 \pm .65$ & $.000^{* *}$ & .63 \\
Apoyo social y familiar & $4.3 \pm .83$ & $4.2 \pm .97$ & .132 & \\
Exigencia de la carrera & $3.3 \pm .79$ & $3.3 \pm .74$ & .537 & \\
Financiamiento y formación & $3.3 \pm .85$ & $3.2 \pm .88$ & .394 & \\
Protestas estudiantiles & $1.9 \pm .85$ & $2.9 \pm 1,17$ & $.000^{* *}$ & .95 \\
Participación y nivelación & $2.5 \pm 1.03$ & $2.7 \pm .94$ & $.009^{* *}$ & .20 \\
Técnicas de estudio & $2.2 \pm 1.16$ & $2.3 \pm 1.21$ & .414 & \\
d=tamaño del efecto & & & & \\
*Diferencias significativas al nivel .05 & & & \\
**Diferencias significativas al nivel .01 & &
\end{tabular}

\section{Discusión}

La escala de Percepción de Flores, et al. (2019) hacia la carrera de Educación Física se basa en las razones en que los estudiantes han indicado como motivos para desertar de la carrera, por ende, los valores más bajos (promedios por dimensiones) que se han encontrado en esta investigación se convierten en factores determinantes para mejorar en las instituciones de educación superior para retener a sus estudiantes, en este sentido, el que posee mayor impacto tiene relación en la comparación de las universidades estatales y privadas en la dimensión Protestas estudiantiles con un $p=.000$ y $d=.95$ y en segundo lugar Gestión universitaria con un $p=.000$ y $d=.63$, esto puede estar dado porque los estudiantes de las universidades estatales son más críticos y con ello más exigentes en sus juicios, también considerando que éstas instituciones tienden a parecer más burocráticas, debido a que responden a otros niveles de gestión (controlaría interna o general de la república) y en cambio las universidades privadas no tienen que pasar por estos procesos y por ende pueden entregar soluciones más eficientes y funcionales en relación a las demandas de sus estudiantes, esto coincide con un estudio realizado por el Departamento de Economía de la Universidad de Chile (2008), pero comparando las respuestas de los encargados de entidades educativas de educación superior (pregrado y postgrado), donde los directivos de las instituciones pertenecientes al Consejo de Rectores de las Universidades Chilenas son más críticos en comparación a los directivos de las instituciones privadas y reconocen debilidades en las metodologías de enseñanza y aprendizaje, además que las carreras no satisfacen las expectativas de los estudiantes.

En relación a las protestas estudiantiles, se puede mencionar que las universidades estatales poseen una misión y visión más social a través de los propios docentes y otras actividades académicas que se desarrollan en el ambiente universitario, en consecuencia, esto provoca que estas expresiones sean más propias de sus vidas involucrándose más en las demandas de aquellos que participan en las protestas y finalmente a los estudiantes les complica más retomar las actividades académicas, debido a este compromiso ideológico.

Por lo tanto, donde se debe prestar mayor énfasis para mejorar las percepciones institucionales de los estudiantes de Educación Física de las universidades públicas son: a) Invitar al diálogo de los estudiantes con los docentes para evitar las protestas estudiantiles y a su vez, continuar con esta predisposición para evitar que los paros no sean tan extensos y luego se estresen (tanto a docentes como alumnos) con evaluaciones concentradas en una fecha; b) En relación a la gestión, se puede mencionar que los estudiantes se preocupan mucho de la calidad docente, organización, los programas y los compromisos (con ello la mejora en la infraestructura de la institución), por ende, estar constantemente realizando grupos de conversación y aplicando encuestas para poseer un seguimiento sobre las inquietudes y solicitudes de los estudiantes, y no tan sólo con los centros de alumnos, pues generalmente los que no forman parte de él (según este estudio) no se sienten partícipes en las decisiones ni demandas; c) En cuanto a la dimensión Apoyo 
social y familiar que se refiere a la valoración social de la carrera también podría significar un motivo para desertar, esto coincide con otros estudios donde existe presión familiar en relación a la desvaloración de estudiar pedagogía (Mizala, Hernández y Makovec, 2011) por los bajos ingresos económicos al momento de insertarse en el mundo laboral.

Por otro lado, también se encontraron diferencias significativas al comparar la percepción institucional de los estudiantes de pedagogía en Educación Física con las variables sociodemográficas, no obstante, no hubo incidencia en el tamaño del efecto, pero a pesar de ello se pueden recomendar lo siguiente: Prestar mayor apoyo y atención a las estudiantes de sexo femenino, pues según los resultados de esta investigación, son las que mayormente se estresan tras las protestas estudiantiles, este resultado coincide con otras investigaciones cuando se han comparado el nivel de estrés entre mujeres y hombres a nivel universitario en relación a la carga académica (Feldman, Goncalves, Chacón, Zaragosa, Bagés, y De Pablo, 2008; Icart, Pulpón e Isern, 2006; JerezMendoza y Oyarzo-Barría, 2015; Marty, Lavín, Figueroa, Larraín y Cruz, 2005).

En relación a las técnicas de estudio, se deben concentrar los esfuerzos en primer año, sobre todo a los estudiantes que no han tenido estudios previos (en educación superior), de origen de establecimientos educacionales municipales y con ingresos socioeconómicos bajos, sin embargo, esta remedial se vuelve complejo cuando existen propuestas de tipo voluntario, ya que, existe baja asistencia lo que dificulta los resultados esperados, por lo tanto, estos deben ser constantes y tener un grado de obligatoriedad (de preferencia en las mallas académicas de las asignaturas o contenidos de ellas). De esta forma se conseguiría que los alumnos lograsen también identificarse con la universidad para generar un sentido de pertenencia, integración y compromiso que, en definitiva, tendrá repercusiones positivas en las percepciones de los estudiantes de la institución (Pascarella y Chaprnan, 1983).

En cuanto a la dimensión Financiamiento y formación, claramente (cómo lo indican la mayoría de las investigaciones como la de Mizala, et al., 2011) se deben generar becas y espacios para que los y las estudiantes puedan acceder o encontrar un medio laboral que permita financiar su carrera académica.

Finalmente, aunque existen escasos estudios en esta población de educación física es relevante seguir estudiando este fenómeno porque los hallazgos podrían contribuir a mejorar las políticas de aseguramiento de la calidad.

\section{Conclusión}

Los resultados revelan que la percepción institucional en la muestra de estudiantes de pedagogía en educación física que se consideran más relevantes son los factores que tienen relación con la Gestión académica y las Protestas estudiantiles, por ende, todos o gran parte de las autoridades de las universidades deben concentrar sus esfuerzos en mejorar dichas valoraciones en su comunidad académica.

Son necesarias investigaciones revisando las percepciones institucionales de los estudiantes de pedagogía en educación física y relacionarlo con otras variables cómo ren- dimiento académico, niveles de estrés, motivación por el estudio, entre otros. También sería interesante realizar un estudio longitudinal para ver como las dimensiones estudiadas en esta investigación van variando en los mismos sujetos a través de los años de formación académica.

\section{Referencias}

Alvarado, E., Luyando, J. \& Picazzo, E. (2015). Percepción de los estudiantes sobre la calidad de las universidades privadas en Monterrey. Revista Iberoamericana de Educación Superior, 17(6), 58-76.

Bustamante, M., Lapo, M., Oyarzún, C.\& Campos, R. (2017). Análisis de la percepción del docente en tres universidades chilenas tras la implementación del Currículum Basado en Competencias. Formación Universitaria, 10(4), 97-110.

Camisón, Z., Gil, E. \& Roca, P. (1999). Hacia modelos de calidad de servicio orientados al cliente en las universidades públicas: el caso de la Universitat Jaume I. Investigaciones Europeas de Dirección y Economía de la Empresa, 5(2), 69-92.

Capelleras, J. \& Veciana, J. (2004). Calidad de servicio en la enseñanza universitaria: desarrollo y validación de una escala de medida. Revista Europea de Dirección y Economía de la Empresa, 13(4), 55-72.

Cea, S., Véliz, C., Aravena, C. \& Maureira, F. (2014). Percepción de los estudiantes de educación física de la UISEK de Chile hacia sus profesores. Cuadernos de Psicología del Deporte, 14(1), 23-28.

Cervantes, V., Peralta, P., Salgado, R., \& Stefanell, I. (2018). Calidad de servicio en una institución de educación superior en la ciudad de Barranquilla. Ciencias Administrativas, 11(6),28-40.

Departamento de Economía de la Universidad de Chile (2008). Informe final: Estudio sobre causas de la deserción universitaria. Disponible en: file:///C:/Users/Adilson/ Downloads/causas-desercion-universitaria-chile.pdf.

Feldman, L., Goncalves, L., Puignau, G., Zaragoza, J., Bagés, N. \& De Paulo, J. (2008). Relaciones entre estrés académico, apoyo social, salud mental y rendimiento académico en estudiantes universitarios venezolanos. Universitas Psychologica, 7(3), 739-752.

Flores, E., Bahamondes, V., Maureira, F. \& González, P. (2019). Motivos de deserción universitaria de estudiantes de educación física de Chile. EmásF, Revista Digital de Educación Física, 10(57), 14-23.

García, I. (2019). Impacto de la vocación sobre la construcción de la identidad profesional en los estudiantes de Magisterio de la mención de Educación Física. TRANCES, Extra 1, 771-784.

Gutiérrez, M., Domenéc, P. \& Torres, E. (2007). Perfil de la educación física y sus profesores desde el punto de vista del alumno. Revista Internacional de Ciencias del Deporte, 8, 39-52.

Icart, M., Pulpón, M. \& Isern, I. (2006). Trastornos menores de salud en una muestra de estudiantes de la Universidad de Barcelona. Educación Médica, 9(3), 42-47.

Jerez-Mendoza, M. \& Oyarzo-Barría, C. (2015). Estrés académico en estudiantes del Departamento de Salud de la Universidad de Los Lagos Osorno. Revista Chilena de Neuro-Psiquiatría, 53(3), 149-157.

López, S. (2007). Evaluación institucional y factores de cambio: La percepción de los académicos de tres universidades del noroeste de México. Revista de la educación superior, 36(144), 7-22.

Marty, M., Lavín, G., Figueroa, M. \& Cruz, M. (2005). Prevalencia de estrés en estudiantes del área de la salud de la Universidad de los Andes y su relación con enfermedades infecciosas. Revista Chilena de Neuro-Psiquiatría, 43(1), 25-32.

Maureira, F. \& Flores, E. (2018). Manual de investigación cuantitativa para estudiantes de educación física. Madrid: Bubok Publishing.

Maureira, F. (2017). Estadística para educación física. Madrid: Bubok Publishing

Mizala, A., Hernández, T. \& Makovec, M. (2011). Determinantes de la elección y deserción en la carrera de pedagogía. Santiago: MINEDUC.

Moreno, A., Sicilia, A. \& Águila, C. (2003). Percepción de los futuros maestros especialistas en educación física sobre su carrera y su futuro profesional. Habilidad Motriz, 16(11), 15-27.

Mujica, F. \& Orellana, N. (2016). Construcción de la Vocación en Estudiantes de Pedagogía en Educación Física: Un Componente Subjetivo de la Formación Profesional. Revista de Educación Física: Renovar la Teoría y Práctica, 143, 18-24.

Pascarella, E. \& D. Chapman, (1983). A Multi-institutional Path Analytical validation of Tinto's Model of College Withdrawal. American Education Research Journal, 20(1), 87-102.

Poblete, F., Linzmayer, L., Matus, C., Garrido, A. \& Flores, C. (2018). Percepción de estudiantes de Pedagogía en Educación Física hacia sus profesores. Retos, 33, 143147

Reyes, O., \& Reyes, M. (2012). Percepcion de la Calidad del Servicio de la Educacion Universitaria de Alumnos y Profesores. Revista internacional administración \& finanzas, 5(5), 87-98.

Sarmiento, D. \& Vinueza, J. (2020). Percepción estudiantil de la calidad del servicio universitario: caso de una Universidad Ecuatoriana, Revista Científica del Amazonas, 2(5), 52-65.

Sotomayor, C., Coloma, C., Parodi, G, Ibáñez, R., Cavada, P. \& Gysling, J. (2013). Percepción de los estudiantes de pedagogía sobre su formación inicial. magis, Revista Internacional de Investigación en Educación, 5(11), 375-392.

Urra, B., Fehrenberg, M., Muñoz, M. Matheu,A. \& Reyno, A. (2019). Teorías implícitas y modelos de formación subyacentes a la percepción de rol del profesor de educación física. Retos, 36, 159-166.

Urra, B., Reyno, A., Fehrenberg, M. \& Muñoz, M (2020). Paradigma educativo y habilidades del profesor asociadas a la percepción de rol docente en Educación Física de estudiantes chilenos. Retos, 37, 362-369. 